\title{
PRIMEIRO ENCONTRO DE BIOMPHALARIA TENAGOPHILA (D'ORBIGNY, 1835) NATURALMENTE INFECTADA, COM SCHISTOSOMA MANSONI, EM ITAJUBÁ, SUL DO ESTADO DE MINAS GERAIS, BRASIL*
}

\author{
Omar dos Santos Carvalho** \\ Cecília Pereira de Souza** \\ Naftale Katz**
}

\begin{abstract}
CARVAlHO, O. dos S. et al. Primeiro encontro de Biomphalaria tenagophila (d'Orbigny, 1835) naturalmente infectada, com Schistosoma mansoni, em Itajubá, sul do Estado de Minas Gerais, Brasil. Rev. Saúde públ., S. Paulo, 19: 88-91, 1985.
\end{abstract}

RESUMO: Foi descrito o primeiro encontro de Blomphalaria tenagophila, naturalmente infectada, em Itajubá, no sul do Estado de Minas Gerais, Brasil. Nas coleções hídricas que cortam a Granja Wenceslau Neto foram coletados 1.501 exemplares de B. tenagophila, dos quais dois $(0,14 \%)$ eliminavam cercárias de $S$. mansoni. Estes dados indicaram que a transmissão da esquistossomose mansoni continua sendo possível nessa cidade, localizando-se, hoje, em Itajubá, o primeiro foco da endemia no sul do Estado de Minas Gerais

UNITERMOS: Biomphalaria tenagophila, infecção. Schistosoma mansoni, ocorrência. Itajubá, Minas Gerais, Brasil.

\section{INTRODUÇÃo}

A cidade de Itajubá, com cerca de 75.000 habitantes, está localizada numa faixa de terra contínua, no sul do Estado de Minas Gerais, adjacente à divisa com o Estado de São Paulo, numa região até há pouco tempo considerada indene para esquistossomose man. soni.

A localidade é banhada pelo rio Sapucaí e dista cerca de $260 \mathrm{~km}$, por rodovia, de São Paulo, SP e $430 \mathrm{~km}$ de Belo Horizonte, MG.

Em algumas coleções hídricas da cidade existe uma densa população de Biomphalaria tenagophila, cujos estudos pioneiros foram realizados por Paraense e Deslandes ${ }^{11}$, em 1955. Posteriormente, foram feitos estudos experimentais com cepas de Schistosoma mansont, utilizando-se aquele planorbídeo (Coelho $^{5}$, 1962; Carvalho ${ }^{2,3}$, 1978, 1979; Carvalho e Souza $\left.{ }^{1}, 1979\right)$.

Recentemente, Carvalho e cols. ${ }^{4}$, em 1981, e Katz e Carvalho ${ }^{6}$, em 1983, reporta. ram os três primeiros casos humanos autóctones de esquistossomose mansoni daquele município e coletaram 1995 exemplares de $B$. tenagophila e 94 de $B$. peregrina, todos negativos para $S$. mansoni.

- Trabalho financiado parcialmente pelo Conselho Nacional de Desenvolvimento Científico e Tecnológico (CNPq) e Financiadora de Estudos e Projetos (FINEP).

* Do Centro de Pesquisas "René Rachou" - FIOCRUZ - Av. Augusto de Lima, 1715 - Caixa Postal 1743 - 30000 - Belo Horizonte, MG - Brasil. 
CARVALHO, O. dos S. et al. Primeiro encontro de Biomphalaria tenagophila (d'Orbigny, 1835) natural. mente infectada, com Schistosoma mansoni, em Itajubá, sul do Estado de Minas Gerais, Brasil. Rev. Saride públ., S. Paulo, 19: 88-91, 1985.

No presente trabalho são apresentados dados sobre a infecção natural de $B$. tenagophila de Itajubá, por $S$. mansont

\section{MATERIAL E MÉTODOS}

No período de $29 / 11$ a $2 / 12 / 83$ realizou-se um levantamento malacológico nas coleções hídricas existentes na Granja Wenceslau Neto, representadas por córregos, poços, brejos e uma pequena lagoa. A granja funciona como escola, abrigando cerca de 86 crianças órfãs.

Foi coletado, com ajuda de pinça e de uma concha metálica perfurada, presa a uma haste de madeira, um grande número de moluscos, os quais foram adequadamente embalados e transportados para laboratório.

Os exemplares coletados foram examinados em microscópio estereoscópico, inicialmente por exposição à luz artificial e posteriormente por compressão entre placas de vidro.

\section{RESULTADOS}

O levantamento malacológico realizado nas diversas coleçð̃es hídricas, existentes na Granja Wenceslau Neto, possibilitou a coleta de 1.501 exemplares de $B$. tenagophila. Não foi encontrada, nesse levantamento, nenhu. ma outra espécie de Biomphalaria.

Quando expostos à luz artificial, $2(0,14 \%)$ dos moluscos capturados eliminaram cercárias de $S$. mansoni. O exame por compressão não revelou nenhum outro exemplar parasitado. Estes 2 exemplares eram originários de uma amostra de 826 moluscos coletados numa área localizada à entrada da referida Granja, náo superior a $2.500 \mathrm{~m}^{2}$. A identificaçđ̃o das cercárias foi feita através de observação morfológica.

\section{DISCUSSÃO}

A distribuição da $B$. tenagophila abrange os Estados das regioes Centro-Oeste, Sudeste e Sul do Brasil. De fato, foi encontrada em
Goiás, Minas Gerais, Espírito Santo, Rio de Janeiro, São Paulo, Paraná, Santa Catarina e Rio Grande do Sul ${ }^{10}$.

A presença da $B$. tenagophila, na cidade de Itajubá, tem sido motivo de preocupação, em função da proximidade do município com a região do Vale Paraíba e da constante movimentação de migrantes, atra1dos pela instalação e expansão do seu parque industrial ${ }^{1,2,3,4,6}$.

Além desses aspectos, deve-se considerar os relatos recentes de exemplares de $B$. tenagophila, naturalmente infectados, em outras localidades do Estado de Minas Gerais. De fato, Melo e cols. 78 , em 1982 e 1983, reportaram o encontro de exemplares de $B$. tenagophila em Jaboticatubas eliminando cercárias de $S$. mansoni. Nessa região, a esquistossomose mansônica é endêmica, e o principal hospedeiro intermediário é a $B$. glabrata.

Posteriormente, Carvalho e cols.* (1984) relataram a presença de $B$. tenagophila, naturalmente infectada, no Lago da Pampulha, em Belo Horizonte.

Em outubro de 1980, dois dos autores do presente trabalho (OSC e NK) constataram a exístência em Itajubá de 3 casos humanos, autóctones, de esquistossomose mansoni. Naquela ocasiø̃o, em reuniøo com autorida. des locais, constituiu-se uma Comissão Central, composta de representantes da Secreta. ria de Saúde do Estado, Prefeitura Municipal, dos serviços da indústria e do comércio, da EMATER e do Exército. Esta Comissáo teria, como atributo, coordenar a execuçăo de um plano de trabalho que deveria ser rea. lizado com urgência e intensidade, na tentativa de evitar a propagação da esquistosso. mose mansoni, na localidade (Katz e Carvalho ${ }^{6}, 1983$ ).

Decorridos 3 anos da visita a Itajubá, e de advertências contidas em trabalhos anteriormente desenvolvidos, na localidade ${ }^{1,2,3,4,6}$, pôde-se, agora, constatar que nenhuma das medidas sugeridas, em outubro de 1980 , foi executada.

- O. dos S. Carvalho e colaboradores. Situaçáo atual da esquistossomose mansoni no Lago da Pampulha, Belo Horizonte, MG, Brasil. Dados inéditos. 
CARVAlHo, O. dos S. et al. Primeiro encontro de Biomphalaria tenagophila (d'Orbigny, 1835) naturalmente infectada, com Schistosoma mansoni, em Itajubá, sul do Estado de Minas Gerais, Brasil. Rev. Saúde públ., S. Paulo, 19: 88-91, 1985.

Por outro lado, o relato que aqui se faz do encontro de exemplares de $B$. tenagophi$l a$, naturalmente infectados, indica que a transmissão do $S$. mansoni continua sendo possivel, localizando-se na cidade de Itajubá, o primeiro foco de esquistossomose mansoni no sul do Estado de Minas Gerais.

\section{AGRADECIMENTOS}

À Diretoria da Faculdade de Medicina de Itajubá pela colaboração e acolhida; aos Srs. Carlos Rubens Teixeira da Silva e Antônio Carlos do Prado do CPqRR/FIOCRUZ, pela assistência técnica.

CARVALHO, O. dos S. et al. [First finding of naturally infected Biomphalaria tenagophila (d'Orivigny, 1835) at Itajubá, South of Minas Gerais State, Brazil]. Revi Saúde públ., S. Paulo, 19:88-91, 1985.

ABSTRACT: The first time the finding of naturally infected Biomphalaria tenagophila at Itajubá in the South of Minas Gerais State, Brazil, was reported. From 1,501 B. tenegophila collected in streams inside the Granja Wenceslau Neto, two snails $(0.14 \%)$ were shedding $S$. mansoni cercariae. These data suggested the possibility of transmition of $S$. mansoni infection in the region of Itajuba, where the first focus of schistosomiasis was found, in the South of Minas Gerais State.

UNITERMS: Biomphalaria tenagophila, infection. Schistosome mansoni, occurrence. Itajubá, Minas Gerais State, Brazil.

\section{REFERENCIAS BIBLIOGRAFICAS}

1. CARVALHO, O. dos S. \& SOUZA, C.P. Comportamento de Biomphalaria tenagophila (d'Orbigny, 1835) de Itajubá (MG, Brasil) exposta à cepa "SJ" de Schistosoma man. soni Sambon, 1907. In: Congresso da Sociedade Brasileira de Parasitologia, 4\%, Campinas, SP, 1979. Campinas, 1979. p. $1-140$.

2. CARVALHO, O. dos S. et al. Infecção experimental de Biomphalarta tenagophila (d'Orbigny, 1835) de Itajubá (MG, Brasil), à cepa "LE" de Schistosoma mansoni Sambon, 1907. In: Congresso da Sociedade Brasileira de Medicina Tropical, 14\%/Congresso da Sociedade Brasileira de Parasitologia, 30, João Pessoa, 1978. João Pessoa, 1978. p. 1-51.

3. CARVAlHO, O. dos S. et al. Suscetibilidade de Biomphalaria tenagophila (d'Orbigny, 1835), de Itajubá (MG), à infecção pela cepa "LE" de Schistosoma mansoni Sambon, 1907, de Belo Horizonte, MG (Brasil). Rev. Saúde públ., S. Paulo, 13:20-5, 1979.

4. CARVALHO, O. dos S. et al. Introdução re cente de esquistosomose no sul de Minas Gerais. I. Itajubá - Estudo de caso. In: Congresso da Sociedade Brasileira de Medicina Tropical, 179, Caldas Novas, GO, 1981. Caldas Novas, GO, 1981. p. 1-213.
5. COELHO, M.V. Suscetibilidade de Australorbis tenagophilus à infecção por Schis. tosome mansoni Rev. Inst. Med. trop. S. Paulo, 5: 289-95, 1962.

6. KATZ, N. \& CARVALHO, O. dos S. Introdução recente de esquistossomose mansoni no sul do Estado de Minas Gerais, Brasil. Mem. Inst. Oswaldo Cruz, 78:281-4, 1983.

7. MELO, A.L. et al. Sobre o encontro de Biomphalaria tenagophila naturalmente in. fectada com Schistosoma mansoni no município de Jaboticatubas. Minas Gerais. In: Congresso da Sociedade Brasileira de Parasitologia, 79, Porto Alegre, 1982. Porto Alegre, 1982. p. 1-180.

8. MELO, A.L. et al. Infecção experimental persistente de Biomphalaria tenogophila pelo Schtstosoma mansont em Jaboticatubas, Minas Gerais. In: Congresso da Federação Latinoamericana de Parasitologia, 6\%/ Congresso da Sociedade Brasileira de Parasitologia, 8\%/Jornada Paulista de Parasitologia, 5ạ, São Paulo, 1983. São Paulo, 1983. p. 1-298.

9. PARAENSE, W.L. Biomphalaria occidentalis sp. n from South America (Mollusca Basommatophora Pulmonata). Mem Inst. Oswaldo Cruz, 76: 199-211, 1981. 
CARVALHO, O. dos S. et al. Primeiro encontro de Biomphalaria tenagophtla (d'Orbigny, 1835) naturalmente infectada, com Schistosoma mansoni, em Itajubá, sul do Estado de Minas Gerais, Brasil. Rev. Saúde públ., S. Paulo, 19: 88-91, 1985.

10. PARAENSE, W.L. DESLANDES, N. Observations on the morphology of Australorbts nigricans Mem. Inst. Oswaldo Cruz, 53: Recebido pare publicacto em 18/04/1984 121-34, 1955.

\section{Resumos de Livros/Book Reviews}

Metodologia Científica para a Area de Saúde, por Sonia Vieira, Campinas, Ed. Sarvier, 1984. 98 p.

É um livro de fácil leitura, que aborda com bastante propriedade e clareza aspectos importantes da metodologia científica.

Os assuntos são apresentados de forma atraente, com exemplos bem escolhidos. A autora adota uma solução interessante para evitar quebra de continuidade das explicações principais no texto, que é fazer chamadas de rodapé para comentários adicionais. A proposição de exercícios ao fim de cada capítulo e suas soluçס̃es no fim do livro é mais um fator positivo.

Os capítulos sāo os seguintes:
- Introdução à Ciência Experimental;

- Experimentos inteiramente ao acaso;

- Os experimentos em blocos ao acaso;

- Estudos observacionais;

- A comparação de vários grupos;

- A busca de relação entre variáveis;

- Algumas idéias em discussão;

- Um pouco de estatística.

É uma obra recomendável para a biblioteca de todo pesquisador.

José Maria Pacheco de Souza Departamento de Epidemiologia - FSP/USP 